\title{
Radial decoupling of small and large dust grains in the transitional disk RX J1615.3-3255
}

\author{
Robin Kooistra $^{1}$, Inga Kamp ${ }^{1}$, Misato Fukagawa ${ }^{2}$, François Ménard ${ }^{3}$, Munetake Momose ${ }^{4}$, Takashi Tsukagoshi ${ }^{4}$, \\ Tomoyuki Kudo $^{5}$, Nobuhiko Kusakabe ${ }^{6}$, Jun Hashimoto ${ }^{6}$, Lyu Abe ${ }^{7}$, Wolfgang Brandner ${ }^{8}$, Timothy D. Brandt ${ }^{9}$, \\ Joseph C. Carson ${ }^{10}$, Sebastian E. Egner ${ }^{5}$, Markus Feldt ${ }^{8}$, Miwa Goto ${ }^{11}$, Carol A. Grady ${ }^{12,13,14}{ }^{\text {, Olivier Guyon }}{ }^{5}$, \\ Yutaka Hayano ${ }^{5}$, Masahiko Hayashi ${ }^{15}$, Saeko S. Hayashi ${ }^{5}$, Thomas Henning ${ }^{8}$, Klaus W. Hodapp ${ }^{16}$, Miki Ishii ${ }^{15}$, \\ Masanori Iye ${ }^{15}$, Markus Janson ${ }^{17}$, Ryo Kandori ${ }^{15}$, Gillian R. Knapp ${ }^{18}$, Masayuki Kuzuhara ${ }^{19}$, Jungmi Kwon ${ }^{20}$, \\ Taro Matsuo ${ }^{21}$, Michael W. McElwain ${ }^{12}$, Shoken Miyama ${ }^{22}$, Jun-Ichi Morino ${ }^{15}$, Amaya Moro-Martin ${ }^{18,23}$, \\ Tetsuo Nishimura ${ }^{5}$, Tae-Soo Pyo ${ }^{5}$, Eugene Serabyn ${ }^{24}$, Takuya Suenaga ${ }^{15,25}$, Hiroshi Suto ${ }^{15,6}$, Ryuji Suzuki ${ }^{15}$, \\ Yasuhiro H. Takahashi ${ }^{20,15}$, Michihiro Takami ${ }^{26}$, Naruhisa Takato ${ }^{5}$, Hiroshi Terada ${ }^{15}$, Christian Thalmann ${ }^{27}$, \\ Daigo Tomono ${ }^{5}$, Edwin L. Turner ${ }^{18,28}$, Makoto Watanabe ${ }^{29}$, John Wisniewski ${ }^{30}$, Toru Yamada ${ }^{31}$, Hideki Takami ${ }^{15}$, \\ Tomonori Usuda ${ }^{15}$, Motohide Tamura ${ }^{20,15,6}$, Thayne Currie ${ }^{5}$, Eiji Akiyama ${ }^{15}$, Satoshi Mayama ${ }^{32,33}$, \\ Katherine B. Follette ${ }^{28}$, and Takao Nakagawa ${ }^{34}$ \\ (Affiliations can be found after the references)
}

Received 12 April 2016 / Accepted 30 September 2016

\begin{abstract}
We present $H$-band $(1.6 \mu \mathrm{m})$ scattered light observations of the transitional disk RX J1615.3-3255, located in the $\sim 1$ Myr old Lupus association. From a polarized intensity image, taken with the HiCIAO instrument of the Subaru Telescope, we deduce the position angle and the inclination angle of the disk. The disk is found to extend out to $68 \pm 12 \mathrm{AU}$ in scattered light and no clear structure is observed. Our inner working angle of $24 \mathrm{AU}$ does not allow us to detect a central decrease in intensity similar to that seen at $30 \mathrm{AU}$ in the $880 \mu \mathrm{m}$ continuum observations. We compare the observations with multiple disk models based on the spectral energy distribution (SED) and submm interferometry and find that an inner rim of the outer disk at $30 \mathrm{AU}$ containing small silicate grains produces a polarized intensity signal which is an order of magnitude larger than observed. We show that a model in which the small dust grains extend smoothly into the cavity found for large grains is closer to the actual $H$-band observations. A comparison of models with different dust size distributions suggests that the dust in the disk might have undergone significant processing compared to the interstellar medium.
\end{abstract}

Key words. circumstellar matter - planet-disk interactions - planets and satellites: formation - protoplanetary disks

\section{Introduction}

The definition of transitional disks is heavily debated in the literature. Espaillat et al. (2014) defined transitional disks as objects that exhibit almost no near-IR excess, yet harbor a strong mid- and far-IR excess. The former suggests that the inner regions have been cleared of material, forming a hole in the disk. Strom et al. (1989) suggested that they are a transition stage in the evolution from an optically thick disk extending towards the star into a dispersed low-mass disk. Disks that have a nearIR excess but a dip in the mid-IR emission can be interpreted as a two-disk system with a gap in between (Espaillat et al. 2012). They are sometimes referred to as pre-transitional disks (Espaillat et al. 2007).

A key question for studying these objects is how their inner region is cleared out. Several mechanisms have been proposed for this. As a consequence of viscosity in disks, they are expected to become optically thin as they accrete (Alexander et al. 2014), but this is a slow process. Photoevaporation, where material on the surface of the disk is heated strongly by the UV or X-ray radiation from the central star, can cause an outflow of material from the disk (Alexander et al. 2014). This cuts off the supply of disk material from the outer disk, making it possible to clear out the inner disk as the material quickly accretes onto the star. A substantial sample of transitional disks, however, show inner holes that are too large together with accretion rates that are too high to be explained by photoevaporation alone (Owen et al. 2011). Furthermore, disk winds driven by magnetohydrodynamic turbulance can also play an important role in the dispersal of disks (Suzuki \& Inutsuka 2009). It is also possible to create gaps in the disk by dynamical interaction with single (e.g., Pinilla et al. 2012; de Juan Ovelar et al. 2013) or multiple massive objects (e.g., Zhu et al. 2011; Dodson-Robinson \& Salyk 2011; Dong et al. 2015). The main candidate for this type of clearing is a planetary body that carves a hole by sweeping up material as it moves through the disk. The transitional disks are thought to be an important stage in understanding the formation of these planets, and several promising canditates for planets within disks have been observed, e.g., HD 100546 (Quanz et al. 2013; Currie et al. 2015), HD 169142 (Reggiani et al. 2014), and LkCa 15 (Kraus \& Ireland 2012; Sallum et al. 2015).

A significant sample of transitional disks are shown to have a cavity in the submm (Andrews et al. 2011). Recently however, comparisons of high-resolution near-IR observations with submm images have shown a possible number of transitional disks with near-IR emission extending into the submm cavity (e.g., Dong et al. 2012), which suggests a decoupling of the distribution of both small and large dust grains. In some cases 
the cavities at both wavelengths have been spatially resolved, indeed showing that the small dust grains can move in closer to the star than the large dust grains (e.g., Muto et al. 2012; Garufi et al. 2013; Grady et al. 2013; Follette et al. 2013).

The transitional disk RX J1615.3-3255 (from here on referred to as RXJ1615) was first detected by Henize (1976). RXJ1615 is located in the constellation Lupus and has been kinematically tied to a young ( $\sim 1$ Myr old) subgroup of the Lupus association at a distance of 185 pc (Makarov 2007). It was identified as a weak-line T Tauri star by Krautter et al. (1997), based on optical spectroscopy. Spitzer IR observations, showed the first evidence of an inner hole in the disk by performing a fit to the SED, designating it a transitional disk (Merín et al. 2010).

Andrews et al. (2011) performed high-resolution $880 \mu \mathrm{m}$ observations and created a disk model that fits both the spectral energy distribution (SED) and their visibilities. Their data shows a decrease in the intensity close to the center, a low density cavity out to a radius of $30 \mathrm{AU}$. The disk is relatively flat and massive, $\sim 12 \%$ of the stellar mass. Because of the large size of the gap and the disk mass, they find that the disk is most likely cleared dynamically by tidal interactions with a low-mass brown dwarf or giant planet companion on a long-period orbit.

In this paper, we present new $H$-band scattered light images of RXJ1615 and reproduce the disk model from Andrews et al. (2011) based on submm interferometry and the SED in order to study the spatial distribution of the large versus small dust grains in this disk.

\section{Observations and data reduction}

Our observations were obtained with the HiCIAO instrument (Tamura et al. 2006) of the Subaru telescope as part of the 16th run of the Strategic Explorations of Exoplanets and Disks with Subaru (SEEDS) survey on July 5, 2012. The images were taken in the $H$-band $(1.6 \mu \mathrm{m})$ using a combination of the HiCIAO quad Polarimetric Differential Imaging (qPDI) and Angular Differential Imaging (ADI) modes. In order to explore structures close to the star, no coronographic mask was used. A summary of the observations and the details of the data are given in Table 1.

\subsection{Data reduction}

We performed the data reduction following the standard description for handling PDI data to obtain the Stokes parameters using cycles of four different angular positions of the Wollaston prism (Hinkley et al. 2009). All images were destriped and corrected for warm and bad pixels, distortion, and instrumental polarization. The images were then derotated to account for the ADI observation mode and photometrically calibrated using data of the standard star HD 203856 observed in the open use program on July 5, 2012.

\subsection{Bad images}

During the data reduction process, we noticed that the quality between exposures varied. This is evidenced in Fig. 1, where we show the FWHM of the stellar light after the distortion correction step. Although the atmospheric attenuation during the night should not have been a problem, the seeing did get above $1^{\prime \prime}$ for a large part of the night. The adaptive optics or not easily able to correct for this, which might explain the variation in the point spread function (PSF) of the exposures. In order to try to resolve the cavity found by Andrews et al. (2011), we wanted to keep
Table 1. Summary of the observations and the obtained data.

\begin{tabular}{|c|c|c|c|}
\hline \multicolumn{4}{|l|}{ Object details } \\
\hline Name & \multicolumn{3}{|c|}{ RX J1615.3-3255 } \\
\hline$\alpha[\mathrm{J} 2000]$ & \multicolumn{3}{|l|}{161520.20} \\
\hline$\delta[\mathbf{J} 2000]$ & \multicolumn{3}{|l|}{-325505.1} \\
\hline Region & \multicolumn{3}{|c|}{ Lupus ( 1 Myr) } \\
\hline Distance & \multicolumn{3}{|l|}{$185 \mathrm{pc}$} \\
\hline Spectral type & \multicolumn{3}{|l|}{$\mathrm{K} 5$} \\
\hline \multicolumn{4}{|l|}{ Observation conditions } \\
\hline Telescope, instrument & \multicolumn{3}{|c|}{ Subaru, HiCIAO } \\
\hline Observation modes & \multicolumn{3}{|l|}{ qPDI \& ADI } \\
\hline Observed band & \multicolumn{3}{|c|}{$H$-band $(1.6 \mu \mathrm{m})$} \\
\hline Diffraction limit & \multicolumn{3}{|l|}{$0.05 \operatorname{arcsec}$} \\
\hline Pixel scale & \multicolumn{3}{|c|}{$9.5 \times 10^{-3} \operatorname{arcsec}$} \\
\hline Standard star & \multicolumn{3}{|c|}{ HD 203856} \\
\hline$R$-band mag observed & \multicolumn{3}{|l|}{11.6} \\
\hline Seeing & \multicolumn{3}{|l|}{$>1^{\prime \prime}$} \\
\hline \multicolumn{4}{|l|}{ Data } \\
\hline \multirow[t]{2}{*}{ Final integration time } & \multicolumn{3}{|l|}{$7.5 \mathrm{~min}$} \\
\hline & Date & $N_{\text {exp }}$ & $t_{\exp }(\mathrm{s})$ \\
\hline Star & 2012-Jul.-05 & 68 & 30 \\
\hline Standard star & & 3 & 1.5 \\
\hline Flat field & 2012-May-13 & 9 & 120 \\
\hline Dark & 2012-Jul.-07 & 100 & 40 \\
\hline
\end{tabular}

Notes. $N_{\exp }$ is the number of exposures and $t_{\exp }$ is the exposure time for each exposure.

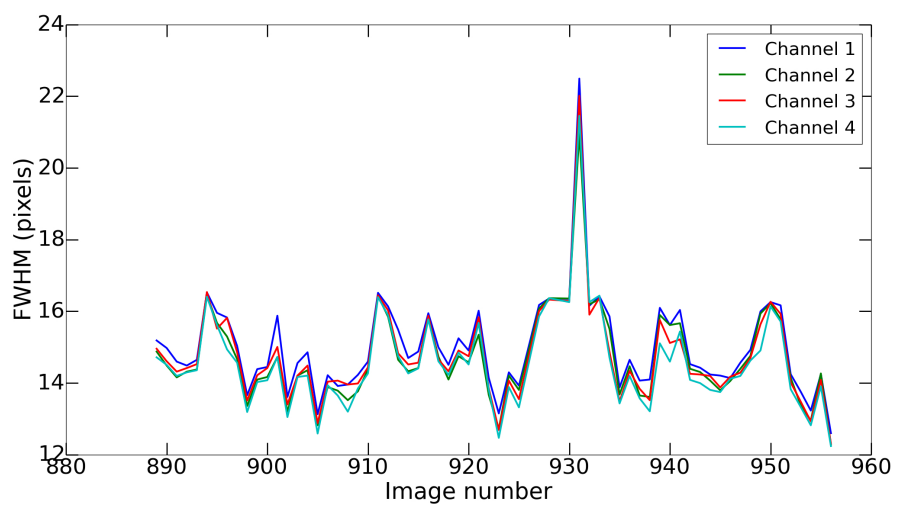

Fig. 1. FWHM in different images. Each line represents a different qPDI channel.

our inner working angle as small as possible. We therefore removed all data where the PSF FWHM is larger than 16 pixels $(0.152 \mathrm{arcsec})$. This effectively meant losing half of the data, bringing the integration time down from $7.5 \mathrm{~min}$ (15 waveplate cycles) to 4 (8 waveplate cycles), which significantly reduced our signal-to-noise, but decreased our inner working angle from $\sim 33 \mathrm{AU}$ to $24 \mathrm{AU}$.

\section{Results}

Using the Stokes $Q$ and $U$ images obtained from the data, we determined the polarized intensity using

$P I=\sqrt{Q^{2}+U^{2}}$

The resulting polarized intensity map is given in the left panel of Fig. 2, where the brightness profile along the major axis of the disk is given inside the plot. Power-law fits to both sides are 




Fig. 2. Polarized intensity map (left) and signal-to-noise map (right). The red contours show the $S / N>3$ region, the green cross denotes the stellar position and the magenta circle gives the inner working angle of $33 \mathrm{AU}$. The region inside the inner working angle is masked. The plot gives the brightness profile along the disk major axis to which we fitted power-law profiles (black lines). The fitted power-law exponents are given in the plot with $1 \sigma$ uncertainties.

Table 2. Physical parameters measured using an ellipsoid fit.

\begin{tabular}{ll}
\hline \hline Parameter (units) & Value \\
\hline PA (deg) $)^{a}$ & $142 \pm 1$ \\
$i(\mathrm{deg})^{b}$ & $52.91 \pm 0.02$ \\
$R_{\text {out }}(\mathrm{AU})$ & $68 \pm 12$ \\
\hline
\end{tabular}

Notes. We give $1 \sigma$ errors. ${ }^{(a)}$ Major axis, measured East from the North. (b) $0^{\circ}$ is face-on.

shown in the plot as black lines. The disk has a relatively shallow radial profile along the major axis, with power-law indices of $-1.17 \pm 0.09$ and $-1.4 \pm 0.1$, compared to the range of -1.7 to -5 found in other disks (Kusakabe et al. 2012; Muto et al. 2012). As a conservative estimate for the errors on the polarized intensity we used the standard deviation between images of different waveplate cycles, divided by the square root of the number of images. Dividing the polarized intensity by the error then gives the signal-to-noise map shown in the right panel of Fig. 2. As can be seen in both figures, we clearly detected the extended disk emission with a signal-to-noise of $\sim 2-6$.

From the polarized intensity image we estimated a few physical parameters of the RXJ1615 disk and summarize them in Table 2. For the position angle (PA), we fitted an ellipsoid using the Image Reduction and Analysis Facility (IRAF ${ }^{1}$ ) package. Assuming an infinitely flat disk, we obtained the inclination angle $(i)$ from the ratio of the major and minor axis of the disk. The uncertainties in these parameters are determined by the IRAF ellipsoid fitting routine. We defined the outer radius $\left(R_{\text {out }}\right)$ as the distance from the stellar position along the major axis at which the $\mathrm{S} / \mathrm{N}$ drops below 3 . We note that the outer radius of $68 \pm 12 \mathrm{AU}$ is smaller than was found from the submm

1 IRAF is distributed by National Optical Astronomy Observatory, which is operated by the Association of Universities for Research in Astronomy, Inc., under cooperative agreement with the National Science Foundation. observations of Andrews et al. (2011; 115 AU). At large distances, the low surface brightness of the disk can be expected to disappear into the noise and therefore our measurement is likely underestimating the true outer radius of the disk. Our inner working angle of $33 \mathrm{AU}$ does not allow for the exploration of the inner 30 AU cavity. However, when decreasing the inner working angle to 24 AU by removing the bad images, as described in Sect. 2.2, we still do not see signs of a depletion in the inner $30 \mathrm{AU}$ of the disk. Although the large dust grains $(\sim \mathrm{mm}$ size $)$ are depleted in the gap (as evidenced by the submm data), this could signify that the small dust grains ( $\lesssim 1 \mu \mathrm{m}$ size $)$ either still survive or that they have a smaller cavity size than the large grains. The latter was also seen in other transitional disks, e.g., SAO 206462 (Muto et al. 2012; Garufi et al. 2013), MWC 758 (Grady et al. 2013), and SR21 (Follette et al. 2013). However, our inner working angle of $24 \mathrm{AU}$ is too close to the 30 AU cavity radius of Andrews et al. (2011) to be certain from this dataset.

In order to confirm the nature of the scattered light, it is possible to look at the direction of the polarization vector. The polarization angle is defined as

$\alpha=\frac{1}{2} \tan ^{-1}\left(\frac{U}{Q}\right)$

We overplot this angle on the polarized intensity in Fig. 3, where the angle of the ticks denotes the polarization angle. In the disk region along the major axis most ticks are aligned in a direction perpendicular to the direction towards the stellar position, which is a clear sign that we are indeed looking at light scattered from the star through the disk. We note, however, that the polarization angle is not aligned perpendicular to the radial direction along the minor axis of the disk, and thus a polarized halo might be affecting the observation data (Hashimoto et al. 2012). This could affect our derivation of the outer radius, position angle and inclination of the disk and could also explain why we find a significantly larger inclination angle than Andrews et al. (2011). 


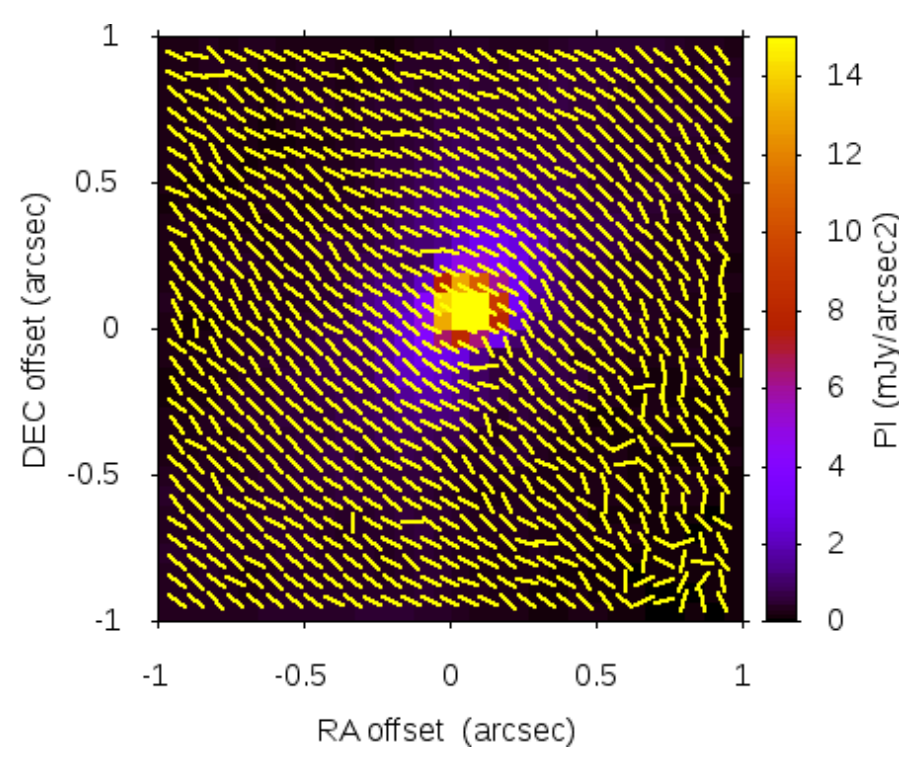

Fig. 3. Polarization angle map overlayed on a block averaged version of PI. The angle of the yellow ticks gives the polarization angle.

\section{Comparison with models}

Andrews et al. (2011) created a disk model of RXJ1615 that fits both the spectral energy distribution (SED) and their observed submm visibilities. In order to see if this same model also agrees with the scattered light observations, we used the Monte Carlo three-dimensional continuum radiative transfer code MCFOST (Pinte et al. 2006) to reproduce the Andrews et al. (2011) disk model and simulate an $H$-band image. The code traces the path of individual packages of photons that propagate through the disk. The photons can undergo scattering, absorption, and reemission events. The main sources of radiation are thermal emission from dust in the disk and photospheric emission from the star. The thermal emission is assumed to be isotropic and depends only on the temperature, density, and opacity of the disk material. The stellar emission is governed by the stellar photospheric spectrum. Photon packages that manage to escape the computation grid are used to calculate the SED and create the simulated $H$-band image.

\subsection{Disk models}

The Andrews et al. (2011) model has three main zones consisting of an inner and outer disk and a puffed up wall at the rim of the outer disk with a gap between the inner and outer disk that has been cleared of material (see Fig. 4).

The density structure of the disk follows a Gaussian profile,

$\rho(r, z)=\rho_{0}(r) \mathrm{e}^{-\frac{z^{2}}{2 H(r)^{2}}}$,

where $H$ denotes the scale height. We assume that the dust follows the same vertical distribution as the gas. The disk flaring is characterized by the flaring exponent $\beta$

$H(r)=H_{0}\left(\frac{r}{r_{0}}\right)^{\beta}$,

where $r_{0}$ is a reference radius and $H_{0}$ the corresponding scale height at that radius. The surface density profile follows either a power-law distribution (inner disk)

$\Sigma(r)=\Sigma_{0}\left(\frac{r}{r_{0}}\right)^{-\epsilon}$

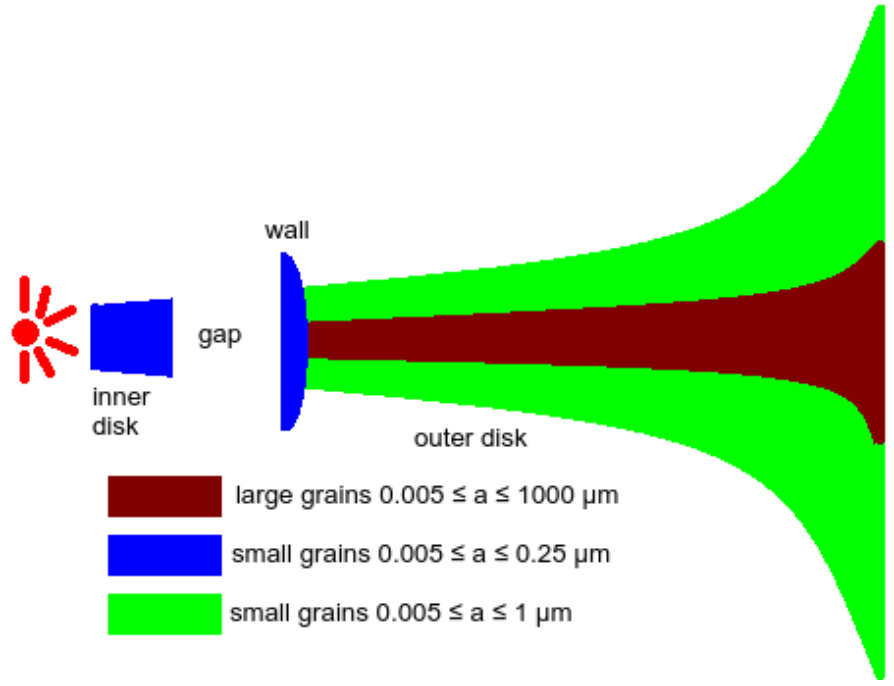

Fig. 4. The Andrews et al. (2011) model.

or a tapered edge distribution (outer disk)

$\Sigma(r)=\Sigma_{\mathrm{c}}\left(\frac{r}{R_{\mathrm{c}}}\right)^{-\epsilon} \mathrm{e}^{-\left(\frac{r}{R_{\mathrm{c}}}\right)^{2-\epsilon}}$

characterized by the surface density exponent $\epsilon$. The parameter $R_{\mathrm{c}}$ in Eq. (6) denotes the characteristic radius at which the exponential term in the distribution becomes important. The dust grain population follows a power-law distribution in grain size ranging from the smallest size $a_{\min }$ to the largest $a_{\max }$ as $n(s) \propto$ $a^{-3.5}$ and we use Draine astronomical silicates (Draine \& Lee 1984).

In the outer disk, we allow for dust settling of the large dust grains onto the midplane of the disk (Dullemond \& Dominik 2004). To mimic this effect, we assume two separate dust populations with different reference scale heights. The flaring index is kept constant throughout the disk. In order to reproduce the SED with MCFOST, we had to tweak some of the parameters adpted by Andrews et al. (2011), e.g., $T_{\text {eff }}$ was decreased by $150 \mathrm{~K}$, the inner disk dust mass was increased by a factor of 10 , and the wall dust mass was decreased by a factor of 100 . The full details of the model are given in Table 3. To investigate the effect of different small dust grain distributions on the produced scattered light, we also explore two other models. One is the same as the Andrews et al. (2011) model but without the wall of intermediate sized dust grains at the inner rim of the outer disk. The other is a thin single disk model that fits the SED (no effort was made to fit details such as the Si feature at 10 micron). In this model the scale height is similar to that of the outer disk in the Andrews et al. (2011) model, but the disk extends closer to the star and small and large dust grains are well mixed throughout the disk. The parameters of this model can also be found in Table 3. All three resulting SEDs are shown in Fig. 5, where the photometric fluxes were de-reddened using the CCM law (Cardelli et al. 1989) with $A_{V}=0.4$ and $R_{V}=5$ (see Table A.1 in the Appendix).

\subsection{Simulated $\mathrm{H}$-band image}

From MCFOST, we get Stokes $Q$ and $U$ images for the three disk models, which we converted to polarized intensity images of the model as it would be seen at a distance of $185 \mathrm{pc}$. Convolving this image with a point spread function (PSF) then allows for a better comparison of the model with the data. There is 
Table 3. Model parameters.

\begin{tabular}{|c|c|c|c|c|c|}
\hline \multicolumn{6}{|l|}{ Stellar parameters } \\
\hline$T_{\text {eff }}$ & \multicolumn{5}{|l|}{$4200 \mathrm{~K}$} \\
\hline$R_{*}$ & \multicolumn{5}{|l|}{$2.16 R_{\odot}$} \\
\hline$M_{*}$ & \multicolumn{5}{|l|}{$1.10 M_{\odot}$} \\
\hline \multicolumn{6}{|l|}{ Disk parameters } \\
\hline \multirow[t]{2}{*}{ Inclination angle } & $41.4^{\circ}$ & & & & \\
\hline & Inner disk & Wall & Outer disk, small grains & Outer disk, large grains & Thin single disk model \\
\hline$\Sigma(r)$ distribution type & Power-law & Power-law & Tapered edge & Tapered edge & Power-law \\
\hline$M_{\text {dust }}\left(M_{\odot}\right)$ & $2.06 \times 10^{-9}$ & $1.10 \times 10^{-8}$ & $1.90 \times 10^{-4}$ & $1.08 \times 10^{-3}$ & $7 \times 10^{-4}$ \\
\hline$H_{100 \mathrm{AU}}(\mathrm{AU})$ & 3.4 & $H_{30 \mathrm{AU}}=2.0$ & 3.4 & 0.68 & 3.5 \\
\hline$R_{\text {in }}(\mathrm{AU})$ & 0.5 & 30 & 30.1 & 30.1 & 2.7 \\
\hline$R_{\text {out }}$ or $R_{\mathrm{c}}(\mathrm{AU})$ & 10 & 30.1 & 115 & 115 & 115 \\
\hline$\beta$ & 1.25 & 1.25 & 1.25 & 1.25 & 1.3 \\
\hline$\epsilon$ & 1 & 1 & 1 & 1 & 1.5 \\
\hline$a_{\min }(\mu \mathrm{m})$ & 0.005 & 0.005 & 0.005 & 0.005 & 0.005 \\
\hline$a_{\max }(\mu \mathrm{m})$ & 0.25 & 0.25 & 1 & 1000 & 1000 \\
\hline
\end{tabular}

Notes. $T_{\text {eff }}$ : effective temperature; $R_{*}$ : stellar radius; $M_{*}$ : stellar mass; $M_{\text {dust }}:$ dust mass; $H_{100 \mathrm{AU}}:$ scale height at $100 \mathrm{AU} ; R_{\text {in }}:$ inner radius; $R_{\text {out }}:$ outer radius; $R_{\mathrm{c}}$ : characteristic radius tapered edge distribution; $\beta$ Flaring exponent; $\epsilon$ : surface density exponent; $a_{\min }$ : minimum size of the dust grains; $a_{\max }:$ maximum size of the dust grains.

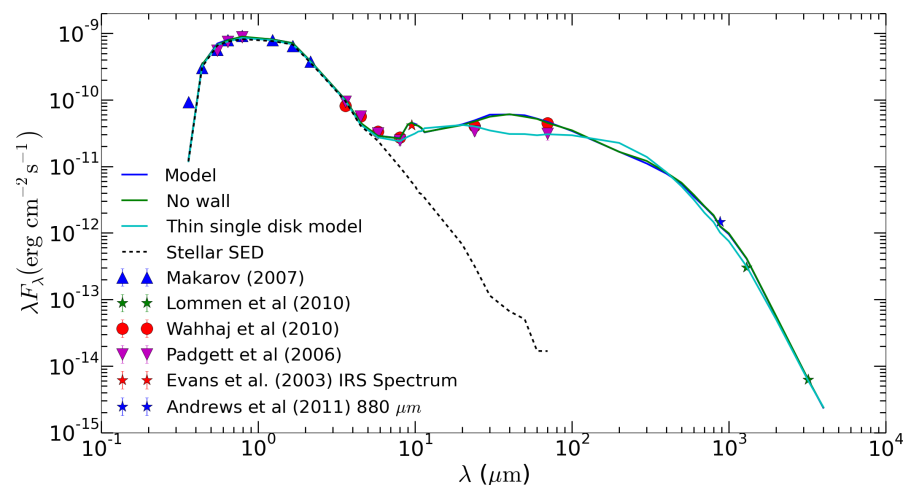

Fig. 5. SED of the disk models. The black dashed line shows the stellar SED from the output of MCFOST. The model parameters are given in Table 3. The references for the photometric data points from the literature are given in the legend. The values of the uncorrected fluxes from the literature can be found in Table A.1.

no data available of a PSF reference star observed on the same night, preventing the development of an accurate PSF model. To still enable a qualitative comparison, we used the polarized intensity profile along the minor axis of the disk from our observations as a PSF estimate. This assumes that the disk is unresolved along the minor axis. We then fitted a double Gaussian to the mean of both sides of this profile (a broad component with FWHM $=0.184$ arcsec and a narrow component with $F W H M=0.039$ arcsec). The resulting convolved $H$-band image of the Andrews et al. (2011) model is shown in Fig. 6.

The extent of the emission is similar to our observations ( $\sim 100 \mathrm{AU})$, but the model clearly produces a much higher signal. This is also evident from the radial profile of the polarized intensity along the major axis of the disk (Fig. 7). Because the wall has a large surface area, it can be expected to produce a lot of scattered light (Thalmann et al. 2010). As can be seen from the radial profile, however, removing the wall only slightly lowers the polarized intensity signal. Instead, most of the emission comes from the inner rim at the edge of the outer disk (30 AU) and is then smeared out by the PSF. The thin single disk model reduces the polarized intensity by a factor of $\sim 2-4$,
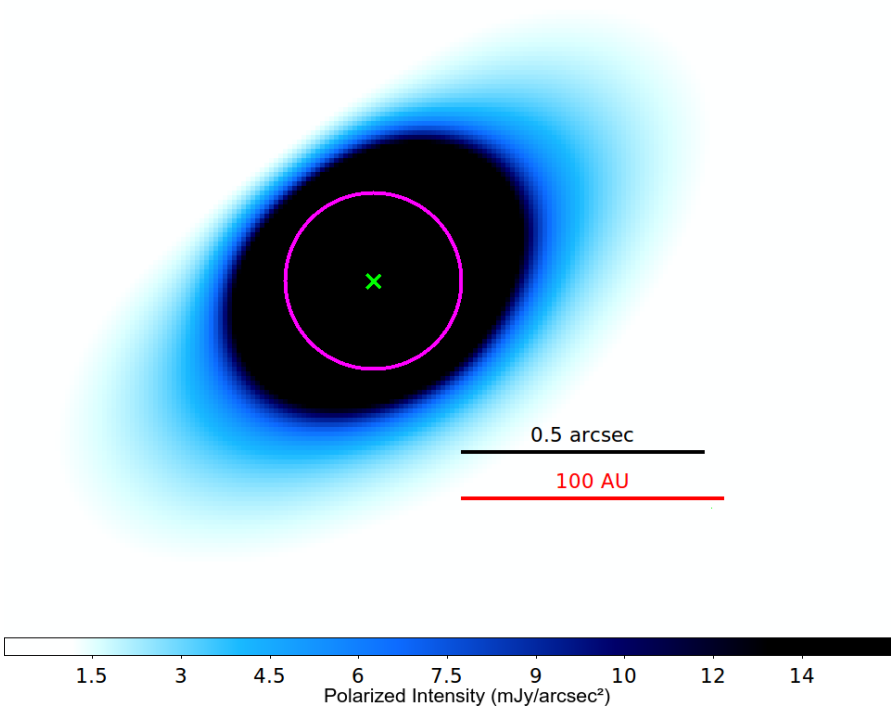

Fig. 6. Simulated $H$-band polarized intensity image of the Andrews et al. (2011) model convolved with a two-component Gaussian PSF with $F W H M=0.183$ arcsec for the broad component and $F W H M=0.038$ arcsec for the narrow one. The scales are identical to Fig. 2 for comparison. The green cross gives the stellar position and the magenta circle denotes the inner working angle from our observations.

bringing it down to a factor of a few from the observed polarized intensity in the outer $\sim 50 \mathrm{AU}$ of the disk. Even though this model has almost the same scale height as the outer disk in the Andrews et al. (2011) model, its inner radius is much smaller (2.7 AU vs. $30 \mathrm{AU}$ ), also making the exposed surface area at the rim smaller ( $H=0.03 \mathrm{AU}$ vs. $0.76 \mathrm{AU})$ and thus reducing the scattered light intensity. This suggests that, although the large grain distribution in RXJ1615 has a cavity out to 30 AU consistent with the $880 \mu \mathrm{m}$ observations, the small dust grains likely extend closer to the star.

However, this does not have to be the only option for bringing down the polarized intensity. A large inner rim much closer 


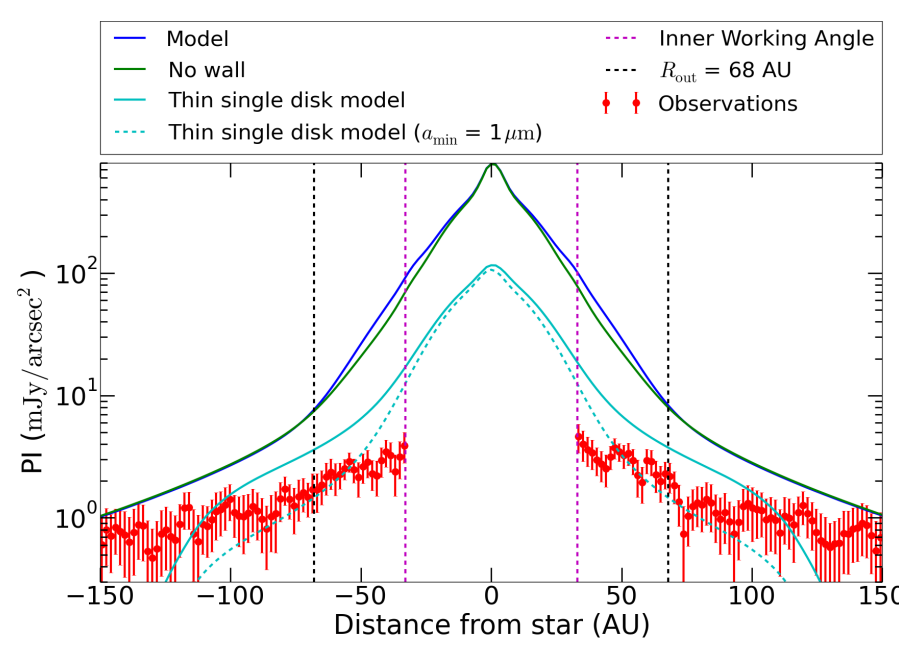

Fig. 7. Radial profile along the major axis of the simulated disk $H$-band PI images for the different models compared to our observations.

to the star could potentially cast a shadow and thus prevent the stellar light from scattering on the disk behind it (Dong et al. 2012). Such a wall would have to be massive enough because, as we can see from our models, most of the light passes directly through the wall and scatters on the rim of the disk behind it instead. A more massive wall would block more stellar light, but would also affect the shape of the SED. Another possibility would be to change the properties of the dust grains (e.g., lowering the grain albedo or different dust type mixtures) or reducing the amount of small particles in the disk. The thin single disk model simply uses astronomical silicate grains with a minimum grain size of $0.005 \mu \mathrm{m}$, which give an albedo of 0.74 and a polarizability of 0.17 . Changing $a_{\min }$ affects both of these quantities. As can be seen from the dashed line in Fig. 7, changing the minimum dust grain size to $a_{\min }=1 \mu \mathrm{m}$ lowers the polarized intensity of the thin single disk model to the same level as we observe. In this case the albedo and the polarizability decrease to 0.63 and 0.12 , respectively. However, when increasing the minimum grain size even further to $3 \mu \mathrm{m}$, the albedo keeps decreasing as expected (0.57), but the polarizability rises again to much higher values $(0.66)$, therefore increasing the disk polarized intensity again above observed values. The current (low) level of polarized intensity suggests that the dust in the disk has already undergone significant processing compared to the ISM. However, more observations at different frequencies and modeling are required before firm conclusions can be drawn.

\section{Conclusion}

We presented the first $H$-band scattered light observations of the transitional disk RX J1615.3-3255. We detected the disk in scattered light, finding an outer radius of $68 \pm 12$ AU. Outside our 24 AU inner working angle, we find no signs of the central depletion in the disk that was previously found in the submm. This could suggest a smaller cavity size for the small grains, but the observations are limited by low signal-to-noise and a large inner working angle. A detailed comparison with several disk models based on fits to the SED and submm visibilities suggests that the small dust grain population is radially decoupled from the large grains. The small dust grains appear to be present closer to the star than the large grains and the dust in the disk has possibly undergone significant processing compared to the ISM. Future higher spatial resolution and higher sensitivity observations (e.g., VLT SPHERE) are required to provide more detailed information on the distribution of the small dust grains in this disk.

Acknowledgements. We thank The Netherlands Foundation for Scientific Research support through the VICI grant 639.043.006. F.Me. acknowledges funding from ANR of France under contract number ANR-16-CE31-0013.

\section{References}

Alexander, R., Pascucci, I., Andrews, S., Armitage, P., \& Cieza, L. 2014, Protostars and Planets VI, 475

Andrews, S. M., Wilner, D. J., Espaillat, C., et al. 2011, ApJ, 732, 42

Bessell, M. S. 1979, PASP, 91, 589

Cardelli, J. A., Clayton, G. C., \& Mathis, J. S. 1989, ApJ, 345, 245

Cohen, M., Wheaton, W. A., \& Megeath, S. T. 2003, AJ, 126, 1090

Currie, T., Cloutier, R., Brittain, S., et al. 2015, ApJ, 814, L27

de Juan Ovelar, M., Min, M., Dominik, C., et al. 2013, A\&A, 560, A111

Dodson-Robinson, S. E., \& Salyk, C. 2011, ApJ, 738, 131

Dong, R., Hashimoto, J., Rafikov, R., et al. 2012, ApJ, 760, 111

Dong, R., Zhu, Z., \& Whitney, B. 2015, ApJ, 809, 93

Draine, B. T., \& Lee, H. M. 1984, ApJ, 285, 89

Dullemond, C. P., \& Dominik, C. 2004, A\&A, 421, 1075

Espaillat, C., Calvet, N., D’Alessio, P., et al. 2007, ApJ, 670, L135 Espaillat, C., Ingleby, L., Hernández, J., et al. 2012, ApJ, 747, 103 Espaillat, C., Muzerolle, J., Najita, J., et al. 2014, Protostars and Planets VI, 497 Evans, II, N. J., Allen, L. E., Blake, G. A., et al. 2003, PASP, 115, 965 Follette, K. B., Tamura, M., Hashimoto, J., et al. 2013, ApJ, 767, 10 Garufi, A., Quanz, S. P., Avenhaus, H., et al. 2013, A\&A, 560, A105 Grady, C. A., Muto, T., Hashimoto, J., et al. 2013, ApJ, 762, 48 Hashimoto, J., Dong, R., Kudo, T., et al. 2012, ApJ, 758, L19 Henize, K. G. 1976, ApJS, 30, 491

Hinkley, S., Oppenheimer, B. R., Soummer, R., et al. 2009, ApJ, 701, 804 Kraus, A. L., \& Ireland, M. J. 2012, ApJ, 745, 5

Krautter, J., Wichmann, R., Schmitt, J. H. M. M., et al. 1997, A\&AS, 123, 329 Kusakabe, N., Grady, C. A., Sitko, M. L., et al. 2012, ApJ, 753, 153

Lommen, D. J. P., van Dishoeck, E. F., Wright, C. M., et al. 2010, A\&A, 515, A77

Makarov, V. V. 2007, ApJ, 658, 480

Merín, B., Brown, J. M., Oliveira, I., et al. 2010, ApJ, 718, 1200

Muto, T., Grady, C. A., Hashimoto, J., et al. 2012, ApJ, 748, L22

Owen, J. E., Ercolano, B., \& Clarke, C. J. 2011, MNRAS, 412, 13

Padgett, D. L., Cieza, L., Stapelfeldt, K. R., et al. 2006, ApJ, 645, 1283

Pinilla, P., Benisty, M., \& Birnstiel, T. 2012, A\&A, 545, A81

Pinte, C., Ménard, F., Duchêne, G., \& Bastien, P. 2006, A\&A, 459, 797

Quanz, S. P., Amara, A., Meyer, M. R., et al. 2013, ApJ, 766, L1

Reggiani, M., Quanz, S. P., Meyer, M. R., et al. 2014, ApJ, 792, L23

Sallum, S., Follette, K. B., Eisner, J. A., et al. 2015, Nature, 527, 342

Strom, K. M., Strom, S. E., Edwards, S., Cabrit, S., \& Skrutskie, M. F. 1989, AJ, 97, 1451

Suzuki, T. K., \& Inutsuka, S.-I. 2009, in AIP Confer. Ser. 1158, eds. T. Usuda, M. Tamura, \& M. Ishii, 161

Tamura, M., Hodapp, K., Takami, H., et al. 2006, in SPIE Conf. Ser., 6269, $62690 \mathrm{~V}$

Thalmann, C., Grady, C. A., Goto, M., et al. 2010, ApJ, 718, L87

Wahhaj, Z., Cieza, L., Koerner, D. W., et al. 2010, ApJ, 724, 835

Zhu, Z., Nelson, R. P., Hartmann, L., Espaillat, C., \& Calvet, N. 2011, ApJ, 729, 47

1 Kapteyn Astronomical Institute, University of Groningen, Postbus 800, 9700 AV Groningen, The Netherlands e-mail: kooistra@astro.rug.nl

2 Division of Particle and Astrophysical Science, Graduate School of Science, Nagoya University, Furo-cho, Chikusa-ku, Nagoya, 464-8602 Aichi, Japan

3 Univ. Grenoble Alpes, CNRS, IPAG, 38000 Grenoble, France

4 College of Science, Ibaraki University, 2-1-1 Bunkyo, Mito, 310-8512 Ibaraki, Japan

5 Subaru Telescope, 650 North A'ohoku Place, Hilo, HI 96720, USA

6 Astrobiology Center of NINS, 2-21-1, Osawa, Mitaka, 181-8588 Tokyo, Japan

7 Laboratoire Lagrange (UMR 7293), Université de Nice-Sophia Antipolis, CNRS, Observatoire de la Côte d'Azur, 28 avenue Valrose, 06108 Nice Cedex 2, France 
8 Max Planck Institute for Astronomy, Königstuhl 17, 69117 Heidelberg, Germany

9 Astrophysics Department, Institute for Advanced Study, Princeton, NJ, USA

10 Department of Physics and Astronomy, College of Charleston, 58 Coming St., Charleston, SC 29424, USA

11 Universitäts-Sternwarte München, Ludwig-MaximiliansUniversität, Scheinerstr. 1, 81679 München, Germany

12 Exoplanets and Stellar Astrophysics Laboratory, Code 667, Goddard Space Flight Center, Greenbelt, MD 20771, USA

13 Eureka Scientific, 2452 Delmer, Suite 100, Oakland, CA 96002, USA

14 Goddard Center for Astrobiology, USA

15 National Astronomical Observatory of Japan, Mitaka, 181-8588 Tokyo, Japan

16 Institute for Astronomy, University of Hawaii, 640 N. A'ohoku Place, Hilo, HI 96720, USA

17 Department of Astronomy, Stockholm University, AlbaNova University Center, 10691 Stockholm, Sweden

18 Department of Astrophysical Science, Princeton University, Peyton Hall, Ivy Lane, Princeton, NJ 08544, USA

19 Department of Earth and Planetary Sciences, Tokyo Institute of Technology, 2-12-1 Ookayama, Meguro-ku, 152-8551 Tokyo, Japan

20 Department of Astronomy, The University of Tokyo, 7-3-1, Hongo, Bunkyo-ku, 113-0033 Tokyo, Japan

21 Department of Earth and Space Science, Graduate School of Science, Osaka University, 1-1 Machikaneyamacho, Toyonaka, 560-0043 Osaka, Japan

22 Hiroshima University, 1-3-2, Kagamiyama, Higashihiroshima, 739-8511 Hiroshima, Japan
${ }^{23}$ Department of Astrophysics, CAB-CSIC/INTA, 28850 Torrejón de Ardoz, Madrid, Spain

24 Jet Propulsion Laboratory, California Institute of Technology, Pasadena, CA 91109, USA

25 Department of Astronomical Science, The Graduate University for Advanced Studies, 2-21-1, Osawa, Mitaka, 181-8588 Tokyo, Japan

26 Institute of Astronomy and Astrophysics, Academia Sinica, PO Box 23-141, Taipei 10617, Taiwan

27 Swiss Federal Institute of Technology (ETH Zurich), Institute for Astronomy, Wolfgang-Pauli-Strasse 27, 8093 Zurich, Switzerland

28 Kavli Institute for Physics and Mathematics of the Universe, The University of Tokyo, 5-1-5, Kashiwanoha, Kashiwa, 277-8568 Chiba, Japan

29 Department of Cosmosciences, Hokkaido University, Kita-ku, Sapporo, 060-0810 Hokkaido, Japan

30 H. L. Dodge Department of Physics \& Astronomy, University of Oklahoma, 440 W Brooks St Norman, OK 73019, USA

31 Astronomical Institute, Tohoku University, Aoba-ku, Sendai, 980-8578 Miyagi, Japan

32 The Center for the Promotion of Integrated Sciences, The Graduate University for Advanced Studies (SOKENDAI),

Shonan International Village, Hayama-cho, Miura-gun, 240-0193 Kanagawa, Japan

33 Department of Astronomical Science, The Graduate University for Advanced Studies (SOKENDAI), 2-21-1 Osawa, Mitaka, 181-8588 Tokyo, Japan

34 Institute of Space and Astronautical Science, Japan Aerospace Exploration Agency, 3-1-1 Yoshinodai, Chuo-ku, Sagamihara, 252-5210 Kanagawa, Japan 


\section{Appendix A: Photometric table}

Table A.1. Photometric data from the literature uncorrected for reddening.

\begin{tabular}{|c|c|c|c|c|c|}
\hline Wavelength $(\mu \mathrm{m})$ & Flux (mJy) & $1 \sigma$ error $(\mathrm{mJy})$ & Magnitude & Zero-magnitude Flux (Jy) & References \\
\hline $0.36(U$-band $)$ & 5.1 & 0.2 & 13.88 & 1810 & 1,7 \\
\hline 0.44 ( $B$-band) & 22.0 & 0.4 & 13.22 & 4260 & 1,7 \\
\hline 0.55 ( $V$-band $)$ & 57.7 & 0.5 & 12.00 & 3640 & 1,7 \\
\hline 0.55 ( $V$-band $)$ & 55.6 & 1.5 & 12.04 & 3640 & 2,7 \\
\hline $0.64\left(R_{\mathrm{c}}\right.$-band $)$ & 101.1 & 0.9 & 11.21 & 3080 & 1,7 \\
\hline $0.64\left(R_{\mathrm{c}}\right.$-band $)$ & 94.7 & 2.6 & 11.28 & 3080 & 2,7 \\
\hline $0.79\left(I_{\mathrm{c}}\right.$-band $)$ & 160.9 & 1.5 & 10.50 & 2550 & 1,7 \\
\hline $0.79\left(I_{\mathrm{c}}\right.$-band $)$ & 155.1 & 4.3 & 10.54 & 2550 & 2,7 \\
\hline $1.235(\mathrm{~J}$-band $)$ & 268.2 & 5.9 & 9.435 & 1594 & 1,8 \\
\hline $1.662(H$-band $)$ & 315.9 & 6.7 & 8.777 & 1024 & 1,8 \\
\hline $2.159\left(K_{\mathrm{s}}\right.$-band $)$ & 251.6 & 4.4 & 8.558 & 666.7 & 1,8 \\
\hline 3.6 & 98.0 & 4.9 & & & 3 \\
\hline 3.6 & 114.0 & 17.1 & & & 2 \\
\hline 4.5 & 85.0 & 4.3 & & & 3 \\
\hline 4.5 & 85.0 & 12.8 & & & 2 \\
\hline 5.8 & 65.0 & 3.3 & & & 3 \\
\hline 5.8 & 61.0 & 9.2 & & & 2 \\
\hline 8.0 & 73.0 & 3.7 & & & 3 \\
\hline 8.0 & 66.0 & 9.9 & & & 2 \\
\hline 9.5 (Spitzer IRS spectrum) & 133.9 & 2.3 & & & 4 \\
\hline 24 & 322.0 & 32.2 & & & 3 \\
\hline 24 & 271.0 & 40.7 & & & 2 \\
\hline 70 & 1049.0 & 167.0 & & & 3 \\
\hline 70 & 727.0 & 145.4 & & & 2 \\
\hline 880 & 430.0 & 2.8 & & & 5 \\
\hline 1300 & 132.0 & 3.9 & & & 6 \\
\hline 3200 & 6.7 & 0.6 & & & 6 \\
\hline
\end{tabular}

Notes. For some data points two references that use different calibration methods are available. We include both values for completeness.

References. (1) Makarov (2007); (2) Padgett et al. (2006); (3) Wahhaj et al. (2010); (4) Evans et al. (2003); (5) Andrews et al. (2011); (6) Lommen et al. (2010); (7) Bessell (1979); (8) Cohen et al. (2003). 